\title{
Linking and coincidence invariants
}

\author{
by \\ Ulrich Koschorke (Siegen)
}

\begin{abstract}
Given a link map $f$ into a manifold of the form $Q=N \times \mathbb{R}$, when can it be deformed to an "unlinked" position (in some sense, e.g. where its components map to disjoint $\mathbb{R}$-levels)? Using the language of normal bordism theory as well as the path space approach of Hatcher and Quinn we define obstructions $\widetilde{\omega}_{\varepsilon}(f), \varepsilon=+$ or $\varepsilon=-$, which often answer this question completely and which, in addition, turn out to distinguish a great number of different link homotopy classes. In certain cases they even allow a complete link homotopy classification.

Our development parallels recent advances in Nielsen coincidence theory and also leads to the notion of Nielsen numbers of link maps.

In the special case when $N$ is a product of spheres sample calculations are carried out. They involve the homotopy theory of spheres and, in particular, James-Hopf invariants.
\end{abstract}

1. Introduction. Throughout this paper let $M_{1}^{m_{1}}, M_{2}^{m_{2}}, N^{n}$ and $Q$ denote smooth manifolds (of the indicated dimensions) without boundary, where $M_{1}, M_{2}$ are compact and $N$ is connected, and let

$$
f=f_{1} \amalg f_{2}: M_{1} \amalg M_{2} \rightarrow Q
$$

be a link map (i.e. the continuous maps $f_{1}$ and $f_{2}$ have disjoint images).

Two such link maps are called link homotopic (compare Milnor [M]) if they can be deformed continuously into each other through a family of link maps.

Question. When can $f$ be unlinked? More precisely: when is $f$ link homotopic to a link map which is trivial in some sense?

In classical link theory two approaches to such problems have played a central role: consider either appropriate intersections or (over)crossings.

2000 Mathematics Subject Classification: Primary 55P35, 55S35, 57Q45, 57R90; Secondary 55M20, 55Q25, 55Q45.

Key words and phrases: unlinking obstruction, link homotopy invariant, overcrossing manifold, normal bordism, path space, Nielsen number. 
If there is some canonical notion of what a "trivial" or "faraway" position of $f_{1}$ should be, and if a homotopy $F_{1}$ moves $f_{1}$ to such a position, measure the intersection locus $C$ of $F_{1}$ with $f_{2}$ in some way. (For instance, if the domain of $f_{1}$ is a sphere and $F_{1}$ is a nulhomotopy this approach leads to the standard procedure of intersecting $f_{2}$ with a singular ball spanned by $f_{1}$.)

If $Q$ has the special product form $Q=N \times \mathbb{R}$ there are natural choices $F_{+}$ and $F_{-}$for such a homotopy: we can move $f_{1}$ in the positive (or negative) $\mathbb{R}$-direction until the whole image of $f_{1}$ lies above (or below) the image of $f_{2}$ with respect to the $\mathbb{R}$-levels. The intersection of such a homotopy with $f_{2}$ corresponds to the overcrossing (or undercrossing, resp.) locus $C_{ \pm}$of the projections $f_{1}^{\prime}$ and $f_{2}^{\prime}$ to $N$.

Whether we base our approach on intersections or over/undercrossings, the resulting unlinking obstruction will be all the more powerful if we register all relevant geometric data concerning the locus $C$ or $C_{ \pm}$as carefully as possible. One rather obvious strategy is to use the language of normal bordism theory. It keeps track of the relations between the stable tangent or normal bundles of the intersection or crossing locus on one hand, and of the manifolds $M_{1}, M_{2}$, and $N$ on the other hand. In very special situations this amounts just to framed bordism (involving stably parallelized manifolds), but in general normal bordism is much more widely applicable and flexible, and a much stronger tool than e.g. oriented bordism (if it applies) or (co)homology with twisted coefficients.

An additional refinement was inspired by the fundamental work of Hatcher and Quinn [HQ]. It is easily overlooked that every coincidence point $x$ comes naturally with a path, namely the constant path at the common value $f_{1}^{\prime}(x)=f_{2}^{\prime}(x)$. But this datum carries very valuable information. Keeping track of it and accommodating our obstruction accordingly in a normal bordism group of a suitable path space will in certain situations supply the necessary data needed to construct a homotopy which deforms maps away from one another or which unlinks link maps.

In Sections 2 and 3 of this paper we define and study unlinking obstructions

$$
\widetilde{\omega}_{+}(f), \widetilde{\omega}_{-}(f) \in \Omega_{m_{1}+m_{2}-n}(E(f) ; \widetilde{\varphi})
$$

which often lead to a complete answer to our original question (see Theorem 2.13 below). They lie in a normal bordism group of an appropriate path space $E(f)$. In many interesting cases this space has an extremely rich topology. For example, already the set $\pi_{0}(E(f))$ of path-components may be huge (there is a natural bijection onto a certain well studied quotient of $\pi_{1}(N)$, the so-called Reidemeister set). The resulting decomposition of our invariants allows us to define Nielsen numbers $N_{+}(f)$ and $N_{-}(f)$ of a link map: just count the (finitely many) essential path-components of $E(f)$, i.e. 
those where the corresponding components of $\widetilde{\omega}_{ \pm}(f)$ are nontrivial. This procedure replaces the often unwieldy $\widetilde{\omega}_{\varepsilon}$-obstruction (which e.g. lies in a group varying with $f$ ) by the numerical link homotopy invariant $N_{\varepsilon}$ which vanishes precisely if $\widetilde{\omega}_{\varepsilon}$ does, $\varepsilon=+$ or - .

A similar point of view was recently introduced into the study of fixed point and coincidence phenomena and led to a coherent Nielsen coincidence theory involving manifolds with arbitrary orientation behaviour and dimension combinations (cf. [K5]).

Our approach is also closely related to recent work of A. Pilz (cf. [P]). His invariant $\alpha_{w}(f)$ registers the decomposition of the bordism class of the intersection $F_{+} \pitchfork f_{2}$ (in framed, oriented or unoriented bordism, $\Omega_{*}^{\mathrm{fr}}, \Omega_{*}$ or $\mathfrak{N}$-as the situation may permit) into components indexed by the Reidemeister set. This is strong enough to yield a link homotopy classification result when $m_{1}+m_{2}=n$ (cf. [P, Theorem 5.4]).

If $M_{1}$ and $M_{2}$ are sufficiently highly connected then natural isomorphisms (exhibited by Hatcher and Quinn) allow us to interpret $\widetilde{\omega}_{ \pm}$itself as a link homotopy invariant. It takes values in the $\left(m_{1}+m_{2}-n\right)$ th framed bordism group of the loop space $\Lambda N$ of $N$.

In particular, this applies when $M_{1}$ and $M_{2}$ are spheres of dimensions $\leq n-2$. In this case there is also a well defined addition of link maps and $\widetilde{\omega}_{ \pm}$turns out to be compatible with this and other natural operations (cf. $5.2-5.4$ below). Moreover a simple construction (using "meridians") supplies many examples of link maps with interesting $\widetilde{\omega}_{ \pm}$-values (cf. 5.7-5.11). Thus our invariants - originally conceived as unlinking obstructions - turn out to also distinguish a great number of different link homotopy classes. In some situations they even lead to a complete classification.

Theorem 1.1. Assume $1 \leq m_{1}+1, m_{2} \leq 2 n-m_{1}-m_{2}-2$ and that $N$ is stably parallelized. Then two base point preserving link maps

$$
f, \widehat{f}: S^{m_{1}} \amalg S^{m_{2}} \rightarrow N \times \mathbb{R}
$$

are link homotopic (in the base point preserving sense) if and only if $\left[f_{i}\right]=$ $\left[\widehat{f}_{i}\right] \in \pi_{m_{i}}(N \times \mathbb{R}), i=1,2$, and if in addition

$$
\widetilde{\omega}_{+}(f)=\widetilde{\omega}_{+}(\widehat{f}) \in \Omega_{m_{1}+m_{2}-n}^{\mathrm{fr}}(\Lambda N)
$$

(or, equivalently, $\left.\widetilde{\omega}_{-}(f)=\widetilde{\omega}_{-}(\widehat{f})\right)$.

This is proved in Section 5; the relation between base point preserving and base point free link homotopy theory is indicated in Remarks 3.9 and 4.2.

For an illustration we consider the case when $N$ is a product of spheres. In Section 4 we reduce the calculation of the framed bordism groups of the loop space $\Lambda N$ (which accommodate $\widetilde{\omega}_{ \pm}(f)$ ) via James-Hopf invariants to 
standard methods of the stable homotopy theory of spheres. This can be used in many concrete settings such as

ExAmple $1.2\left(N=S^{1} \times S^{2} \times S^{8}\right)$. The (base point free) link homotopy class of a link map

$$
f=f_{1} \amalg f_{2}: S^{6} \amalg S^{7} \rightarrow S^{1} \times S^{2} \times S^{8} \times \mathbb{R}
$$

is completely determined by the homotopy classes

$$
\left[f_{1}\right] \in\left[S^{6}, N \times \mathbb{R}\right] \cong \mathbb{Z}_{12}, \quad\left[f_{2}\right] \in\left[S^{7}, N \times \mathbb{R}\right] \cong \mathbb{Z}_{2}
$$

of the component maps and by the (base point free) unlinking obstruction $\left[\widetilde{\omega}_{+}(f)\right]$ which lies in

$$
\Omega_{2}^{\mathrm{fr}}(\Lambda N) / \sim \cong\left(\bigoplus_{-\infty}^{\infty}\left(\Omega_{2}^{\mathrm{fr}} \oplus \Omega_{1}^{\mathrm{fr}} \oplus \Omega_{0}^{\mathrm{fr}}\right)\right) / \sim \cong\left(\mathbb{Z}_{2} \oplus \mathbb{Z}_{2} \oplus \mathbb{Z}\right)\left[X^{ \pm 1}\right] / \sim
$$

Here two (formal) Laurent polynomials (with coefficients in the group $\mathbb{Z}_{2} \oplus$ $\left.\mathbb{Z}_{2} \oplus \mathbb{Z}\right)$ are equivalent if they differ by the factor $X^{j}$ for some integer $j$.

This follows from Theorems 1.1, 4.1, Remarks 3.9, 4.2 and the tables of Toda [T].

ExAmple $1.3\left(N=\left(S^{1}\right)^{3} \times S^{2}\right)$. Both for $\varepsilon=+$ and $\varepsilon=-$ every element of

$$
\Omega_{1}^{\mathrm{fr}}\left(\Lambda\left(\left(S^{1}\right)^{3} \times S^{2}\right)\right) \cong \bigoplus_{j \in \mathbb{Z}^{3}}\left(\mathbb{Z}_{2} \oplus \mathbb{Z}\right)
$$

occurs as the $\widetilde{\omega}_{\varepsilon}$-value of a (base point preserving) link map

$$
f: S^{3} \amalg S^{3} \rightarrow\left(S^{1}\right)^{3} \times S^{2} \times \mathbb{R} .
$$

This and further examples will be discussed at the end of Section 5 .

2. The unlinking obstructions $\widetilde{\omega}_{ \pm}$and $\omega_{ \pm}$. In this section we adapt the coincidence invariants $\widetilde{\omega}$ and $\omega$ constructed in [K5] to the setting of link maps. We obtain the obstructions $\widetilde{\omega}_{\varepsilon}$ and $\omega_{\varepsilon}$ where $\varepsilon$ stands for the symbols + or $-($ and, in formulas, for the factors +1 and -1$)$.

Throughout the remainder of this paper (unless mentioned otherwise)

$$
f=f_{1} \amalg f_{2}: M_{1} \amalg M_{2} \rightarrow N \times \mathbb{R}
$$

is a link map into a manifold of the indicated product form, and $f_{i}=\left(f_{i}^{\prime}, f_{i}^{\prime \prime}\right)$ is the corresponding decomposition via the projections to $N$ and $\mathbb{R}$, resp., $i=1,2$. Consider also the product manifold $M:=M_{1} \times M_{2}$ with the dimension $m:=m_{1}+m_{2}$ and the projections $p_{i}: M \rightarrow M_{i}, i=1,2$. 
Our discussion will center around the space $\left(^{*}\right)$

$$
E(f):=\left\{\left(\left(x_{1}, x_{2}\right) ; \theta\right) \in M \times P(N) \mid \theta(0)=f_{1}^{\prime}\left(x_{1}\right), \theta(1)=f_{2}^{\prime}\left(x_{2}\right)\right\}
$$

where $P(N)$ is the set of all continuous paths $\theta:[0,1] \rightarrow N$, endowed with the compact-open topology. Let pr $: E(f) \rightarrow M$ denote the obvious fiber projection.

If the map

$$
\left(f_{1}^{\prime} \circ p_{1}, f_{2}^{\prime} \circ p_{2}\right)=f_{1}^{\prime} \times f_{2}^{\prime}: M=M_{1} \times M_{2} \rightarrow N \times N
$$

is smooth and transverse to the diagonal

$$
\Delta:=\{(y, y) \in N \times N \mid y \in N\}
$$

then the $\varepsilon$-coincidence locus

$$
C_{\varepsilon}(f):=\left\{\left(x_{1}, x_{2}\right) \in M_{1} \times M_{2} \mid f_{1}^{\prime}\left(x_{1}\right)=f_{2}^{\prime}\left(x_{2}\right), \varepsilon f_{1}^{\prime \prime}\left(x_{1}\right)<\varepsilon f_{2}^{\prime \prime}\left(x_{2}\right)\right\}
$$

(where " $f_{2}$ overcrosses $f_{1}$ " if $\varepsilon=+$ and $f_{2}$ undercrosses $f_{1}$ if $\varepsilon=-$ ) is a closed smooth $(m-n)$-dimensional manifold, equipped with the map

$$
\tilde{g}_{\varepsilon}: C_{\varepsilon}(f) \rightarrow E(f)
$$

which sends $\left(x_{1}, x_{2}\right) \in C_{\varepsilon}(f)$ to $\left(\left(x_{1}, x_{2}\right)\right.$, constant path at $\left.f_{1}^{\prime}\left(x_{1}\right)=f_{2}^{\prime}\left(x_{2}\right)\right)$ and with a stable vector bundle isomorphism

$$
\bar{g}_{\varepsilon}: T C_{\varepsilon}(f) \oplus \widetilde{g}_{\varepsilon}^{*}\left(\operatorname{pr}^{*}\left(\left(f_{1}^{\prime} \circ p_{1}\right)^{*}(T N)\right)\right) \cong \widetilde{g}_{\varepsilon}^{*}\left(\operatorname{pr}^{*}(T M)\right)
$$

(since the normal bundle $\nu\left(C_{\varepsilon}(f), M\right)$ is canonically isomorphic to the bundle $\left.\left(f_{1}^{\prime} \circ p_{1}\right)^{*}(T N)\right)$.

If $f$ is an arbitrary link map, apply this construction to an approximation of $f_{1}^{\prime} \times f_{2}^{\prime}$ which is smooth and transverse to $\Delta$.

In any case the resulting triples $\left(C_{+}, \widetilde{g}_{+}, \bar{g}_{+}\right)$and $\left(C_{-}, \widetilde{g}_{-}, \bar{g}_{-}\right)$determine well defined normal bordism classes

$$
\widetilde{\omega}_{+}(f), \widetilde{\omega}_{-}(f) \in \Omega_{m-n}(E(f) ; \widetilde{\varphi})
$$

as in $[\mathrm{K} 5, \S 4]$; here

$$
\varphi:=\left(f_{1}^{\prime} \circ p_{1}\right)^{*}(T N)-T M, \quad \widetilde{\varphi}:=\operatorname{pr}^{*}(\varphi)
$$

are the relevant (virtual) coefficient bundles over $M$ and $E(f)$, respectively.

Clearly we have

$$
\widetilde{\omega}\left(f_{1}^{\prime} \circ p_{1}, f_{2}^{\prime} \circ p_{2}\right)=\widetilde{\omega}_{+}(f)+\widetilde{\omega}_{-}(f),
$$

where the left hand term is the full coincidence invariant discussed in [K5]. Indeed, all we have done here is to decompose the coincidence locus $\left(f_{1}^{\prime} \times\right.$ $\left.f_{2}^{\prime}\right)^{-1}(\Delta)$ disjointly into its overcrossing and undercrossing parts.

$\left(^{*}\right)$ In $[\mathrm{HQ}]$ (and [K5], resp.) this space is denoted by $E\left(f_{1}^{\prime}, f_{2}^{\prime}\right)$ (and by $E\left(f_{1}^{\prime} \circ p_{1}\right.$, $\left.f_{2}^{\prime} \circ p_{2}\right)$, resp.). 
If we forget the path space aspect of our data and keep track only of the over- or undercrossing manifolds, together with the way they sit in $M$ and with their "twisted framings" $\bar{g}_{\varepsilon}$, we obtain the weaker invariants

$$
\omega_{\varepsilon}(f):=\left[C_{\varepsilon} \text {, inclusion, } \bar{g}_{\varepsilon}\right]=\operatorname{pr}_{*}\left(\widetilde{\omega}_{\varepsilon}(f)\right) \in \Omega_{m+n}(M ; \varphi) .
$$

Definition 2.11. The link map $f$ is $\varepsilon$-unlinked if

$$
\varepsilon\left(f_{1}^{\prime \prime}\left(x_{1}\right)-f_{2}^{\prime \prime}\left(x_{2}\right)\right)>0 \quad \text { for all }\left(x_{1}, x_{2}\right) \in M_{1} \times M_{2}
$$

(compare 2.1) or, equivalently, if the image $f_{1}\left(M_{1}\right)$ in $N \times \mathbb{R}$ lies strictly above (or below, resp.) $f_{2}\left(M_{2}\right)$ with respect to the $\mathbb{R}$-coordinate when $\varepsilon=+$ (or $\varepsilon=-$, resp.).

We say that $f$ is $\varepsilon$-unlinkable if $f$ is link homotopic to an $\varepsilon$-unlinked link map.

Proposition 2.12. If $f$ is $\varepsilon$-unlinkable, then $\widetilde{\omega}_{\varepsilon}(f)=0$ and therefore also $\omega_{\varepsilon}(f)=0$.

Proof. If $f$ is $\varepsilon$-unlinked, then $C_{\varepsilon}(f)=\emptyset$ and hence $\widetilde{\omega}_{\varepsilon}(f)=0$. Moreover, recall from $[\mathrm{K} 5, \S \S 3-4]$ that homotopies of $f_{i}, i=1,2$, induce isomorphisms of normal bordism groups which are compatible with the full coincidence invariants $\widetilde{\omega}$ and - if we are dealing with link homotopies-preserve even the decomposition $\widetilde{\omega}=\widetilde{\omega}_{+}+\widetilde{\omega}_{-}$.

The methods of Hatcher and Quinn yield the following converse result.

Theorem 2.13. Assume that $m_{1}+1, m_{2} \leq 2 n-m_{1}-m_{2}-2$ or $m_{1}, m_{2}+1 \leq 2 n-m_{1}-m_{2}-2$. If after a suitable link homotopy $f_{1}$ and $f_{2}$ project to smooth immersions into $N$ (this holds in particular if $M_{1}, M_{2}$ and $N$ are stably parallelizable), then for $\varepsilon=+$ and $\varepsilon=-$ we have the following: $f$ is $\varepsilon$-unlinkable precisely when $\widetilde{\omega}_{\varepsilon}(f)=0$.

Proof. Our claim is valid for $f$ and $\varepsilon$ if and only if it holds for $f_{2} \amalg f_{1}$ and $-\varepsilon$ (compare the discussion of (4.5) in [K5]). After possibly interchanging $f_{1}$ and $f_{2}$ we may therefore assume the first of the above-mentioned inequalities.

Apply the generalized Whitney trick construction of the proof of Theorem 2.2 in [HQ] to the immersions $F_{1}=F_{\varepsilon}$ (compare 2.14 below, or the beginning of our introduction) and $f_{2}$, as well as to a nulbordism of the over/undercrossing data of $C_{\varepsilon} \approx F_{1} \pitchfork f_{2}$. The resulting deformation will move $F_{1}, f_{2}$ to maps $\widehat{F}_{1}, \widehat{f}_{2}$ with disjoint images. Since the key steps of the construction are based on approximations we can make sure that it does not interfere with $F_{1} \mid\left(M_{1} \times\{0,1\}\right)$ and faraway $\mathbb{R}$-levels. Thus $f$ is link homotopic to $f_{1} \amalg \widehat{f}_{2}$ and, via $\widehat{F}_{1}$, to an $\varepsilon$-unlinked link map.

REMARK 2.14. The previous proof is based on a generally valid alternative interpretation of our invariants. Let

$$
F_{1}=\left(f_{1}^{\prime}, F_{1}^{\prime \prime}\right): M_{1} \times[0,1] \rightarrow N \times \mathbb{R}
$$


be a homotopy which deforms $F_{1}(-, 0)=f_{1}$ monotonically in the positive (or negative) $\mathbb{R}$-direction until $F_{1}(-, 1) \amalg f_{2}$ is $\varepsilon$-unlinked, $\varepsilon=+$ (or $\varepsilon=-$, resp.). Then $F_{1}$ and $f_{2}$ define a pair of maps from $M_{1} \times M_{2} \times[0,1]$ to $N \times \mathbb{R}$ whose coincidence manifold is essentially $C_{\varepsilon}(f)$, with compatible coincidence data. Thus the resulting normal bordism class corresponds to $\varepsilon \widetilde{\omega}_{\varepsilon}(f)$ via the isomorphism induced by the projections $M \times(0,1) \sim M$ and $N \times \mathbb{R} \sim N$.

This alternative ("intersection") approach allows us sometimes to extend our invariants to link maps into more general target manifolds $Q$ (e.g. if $M_{1}=S^{m_{1}}$ and $\left.\pi_{m_{1}}(Q)=\pi_{m_{1}+1}(Q)=0\right)$.

Now consider the special case that $N$ has the form $N=N^{\prime} \times \mathbb{R}$. Then $\widetilde{\omega}\left(f_{1}^{\prime} \circ p_{1}, f_{2}^{\prime} \circ p_{2}\right)=0$ since the extra $\mathbb{R}$-direction allows us to move $f_{1}^{\prime} \circ p_{1}$ and $f_{2}^{\prime} \circ p_{2}$ apart. Thus $\widetilde{\omega}_{+}(f)=-\widetilde{\omega}_{-}(f)$ (cf. 2.9). In fact, we can use the projection along any ray $\mathbb{R}_{+} \cdot v$ in $\mathbb{R} \times \mathbb{R}$ (where $v \neq 0$ ) to study the over/undercrossing behaviour of link maps into $N^{\prime} \times \mathbb{R} \times \mathbb{R}$.

EXAMPLE 2.15 (Classical link maps). Here $M_{i}=S^{m_{i}}, N=\mathbb{R}^{n}, n>0$, and $f: S^{m_{1}} \amalg S^{m_{2}} \rightarrow \mathbb{R}^{n+1}$. Because of the linear structure on $\mathbb{R}^{n+1}$ the fiber projection pr: $E(f) \rightarrow S^{m_{1}} \times S^{m_{2}}$ is a homotopy equivalence so that $\widetilde{\omega}_{+}(f)=-\widetilde{\omega}_{-}(f)$ is precisely as strong as the invariant (cf. 2.10)

$$
\omega_{+}(f) \in \Omega_{m-n}^{\mathrm{fr}}\left(S^{m_{1}} \times S^{m_{2}}\right) \cong \Omega_{m-n}^{\mathrm{fr}} \oplus \Omega_{m_{1}-n}^{\mathrm{fr}} \oplus \Omega_{m_{2}-n}^{\mathrm{fr}} .
$$

In this decomposition we use the isomorphism $\Omega_{*}^{\mathrm{fr}}\left(S^{m_{i}} \times X\right) \cong \Omega_{*}^{\mathrm{fr}}(X) \oplus$ $\Omega_{*-m_{i}}^{\mathrm{fr}}(X)$ defined by the projection to $X$ and by transverse intersection with $\left\{*_{i}\right\} \times X$; furthermore, $\omega_{+}(f)$ corresponds to the triple

$$
\left(\alpha(f), \omega_{+}\left(f \mid\left(S^{m_{1}} \amalg\left\{*_{2}\right\}\right)\right), \omega_{+}\left(f \mid\left(\left\{*_{1}\right\} \amalg S^{m_{2}}\right)\right)\right)
$$

consisting of the "generalized linking number" $\alpha(f)$ (cf. [K2]) and of the overcrossing invariants of one sphere with just the base point of the other sphere.

In the dimension range of Theorem 2.13 the second and third components of this triple vanish; thus $f$ is $\varepsilon$-unlinkable or, equivalently, link nulhomotopic precisely if $\alpha(f)=0$. Actually, N. Habegger and U. Kaiser [HK] have shown that the $\alpha$-invariant classifies $f$ completely up to link homotopy in the more general range $2\left(m_{1}+m_{2}\right) \leq 3 n-2 ; m_{1}, m_{2}<n$. (Compare also [S].)

In contrast to this example we will see below that $\widetilde{\omega}_{\varepsilon}(f)$ is often considerably stronger than $\omega_{\varepsilon}(f)$.

\section{Nielsen numbers of link maps and other link homotopy in-} variants. In order to get a better understanding of our invariants and of the groups in which they lie we need to recall a few facts about the Hurewicz fibration pr : $E(f) \rightarrow M$ (compare $[\mathrm{K} 5, \S 2])$.

Pick points $x_{0}=\left(x_{10}, x_{20}\right) \in M=M_{1} \times M_{2}, y_{0} \in N$ and paths $\sigma_{i}$ in $N$ joining $y_{0}$ to $f_{i}^{\prime}\left(x_{i 0}\right), i=1,2$. This choice determines a homotopy equivalence 
between the space $\Lambda\left(N, y_{0}\right)$ of loops in $N$ starting and ending in $y_{0}$, and the fiber $\mathrm{pr}^{-1}\left(x_{0}\right)$ which consists of all paths in $N$ from $f_{1}^{\prime}\left(x_{10}\right)$ to $f_{2}^{\prime}\left(x_{20}\right)$. We compose with the fiber inclusion to obtain

$$
\text { incl : } \Lambda\left(N, y_{0}\right) \rightarrow E(f) .
$$

Proposition 3.2 (cf. $[\mathrm{K} 5,2.1])$. The induced map

$$
\text { incl }_{*}: \pi_{1}\left(N, y_{0}\right)=\pi_{0}\left(\Lambda\left(N, y_{0}\right)\right) \rightarrow \pi_{0}(E(f))
$$

yields a bijection from the so-called Reidemeister set

$$
R:=\sigma_{1 *} f_{1 *}^{\prime}\left(\pi_{1}\left(M_{1}, x_{10}\right)\right) \backslash \pi_{1}\left(N, y_{0}\right) / \sigma_{2 *} f_{2 *}^{\prime}\left(\pi_{1}\left(M_{2}, x_{20}\right)\right)
$$

onto the set of path-components of $E(f)\left(\right.$ here $\sigma_{i *}: \pi_{1}\left(N, f_{i}^{\prime}\left(x_{i 0}\right)\right) \rightarrow \pi_{1}\left(N, y_{0}\right)$ denotes the obvious isomorphism induced by $\left.\sigma_{i}, i=1,2\right)$.

Thus the rich geometry of $E(f)$ manifests itself already in a possibly very large number of path-components. However, since the coincidence manifold $C_{\varepsilon}$ is compact, only finitely many path-components $A \in \pi_{0}(E(f))$ are essential, i.e. the corresponding direct summand

$$
\widetilde{\omega}_{\varepsilon, A}(f)=\left[C_{\varepsilon, A}(f):=\widetilde{g}^{-1}(A), \widetilde{g}\left|C_{\varepsilon, A}(f), \bar{g}\right|\right]
$$

of

$$
\widetilde{\omega}_{\varepsilon}(f) \in \Omega_{m-n}(E(f) ; \widetilde{\varphi})=\bigoplus_{A \in \pi_{0}(E(f))} \Omega_{m-n}(A ; \widetilde{\varphi} \mid A)
$$

is nonzero.

The (nonnegative, integer) Nielsen number

$$
N_{\varepsilon}(f):=\#\left\{A \in \pi_{0}(E(f)) \mid \widetilde{\omega}_{\varepsilon, A}(f) \neq 0\right\}
$$

counts these essential path-components of $E(f)$ (or, equivalently, the essential Reidemeister classes). This is a refinement of the concept of Nielsen numbers studied in [K5], and we have

$$
N\left(f_{1}^{\prime} \circ p_{1}, f_{2}^{\prime} \circ p_{2}\right) \leq N_{+}(f)+N_{-}(f) .
$$

Clearly $N_{\varepsilon}(f)$ vanishes if and only if $\omega_{\varepsilon}(f)$ does. In other respects the Nielsen number is much cruder than the invariant $\widetilde{\omega}_{\varepsilon}(f)$ which, however, has the drawback that it lies in a group which varies with $f$.

Proposition 3.6. If two link maps are link homotopic, then they have the same Nielsen numbers.

Proof. According to [K5] (see the discussion of 4.4) any homotopy $F$ from $f$ to another link map $\widehat{f}$ yields a homotopy equivalence $E(f) \sim E(\widehat{f})$ and an isomorphism of normal bordism groups which maps the full coincidence invariant $\widetilde{\omega}\left(f_{1}^{\prime} \circ p_{1}, f_{2}^{\prime} \circ p_{2}\right)$ to $\widetilde{\omega}\left(\widehat{f}_{1}^{\prime} \circ p_{1}, \widehat{f}_{2}^{\prime} \circ p_{2}\right)$. If $F$ is a link homotopy this isomorphism preserves also the decomposition $\widetilde{\omega}=\widetilde{\omega}_{+}+\widetilde{\omega}_{-}$. 
The Nielsen number $N_{\varepsilon}(f), \varepsilon=+$ or - , is an example of a link homotopy invariant extracted from $\widetilde{\omega}_{\varepsilon}(f)$ and taking values in a set which is independent of $f$. Another such example is $\omega_{\varepsilon}(f)$ (in the special case where $N$ is stably parallelizable and hence $\varphi=-T M$, cf. 2.10 and 2.8). The invariant $\alpha_{w}(f)$ of Alexander Pilz (cf. [P, 3.9]) can be interpreted as a third such example: assume $f$ preserves base points (e.g. $\left.f_{i}\left(x_{i 0}\right)=\left(y_{0},(-1)^{i}\right), i=1,2\right)$ so that the contributions $\widetilde{\omega}_{\varepsilon, A}(f)$ of the various path-components $A \in \pi_{0}(E(f))$ to $\widetilde{\omega}_{\varepsilon}(f)$ (cf. 3.3 and 3.4) can be parametrized by the Reidemeister set $R$ (cf. 3.2) which remains unchanged by base point preserving homotopies; then

$$
\alpha_{w}(f)=\left\{\left[C_{+, A}(f)\right]\right\} \in \bigoplus_{A \in R} \Omega_{m-n}
$$

where $\Omega_{*}$ stands for framed or (un)oriented bordism according as $M_{1}, M_{2}$ and $N$ are framed or (un)oriented, respectively. Thus $\alpha_{w}(f)$ neglects e.g. the map $\widetilde{g} \mid C_{+, A}(f)$ but still registers the decomposition of the overcrossing locus $C_{+}(f)$ into various "Reidemeister (or Nielsen) classes". If $m=n$, this enables A. Pilz to obtain full classification results (cf. [P, 5.4]).

Next we recall a result of Hatcher and Quinn (cf. [HQ, 3.1]) which allows us sometimes to interpret $\widetilde{\omega}_{\varepsilon}(f)$ itself (without any loss of information) as a link homotopy invariant.

Proposition 3.8. Assume that $M_{1}$ and $M_{2}$ are $(m-n+1)$-connected. Then the map incl (cf. 3.1), together with a choice of an orientation of $\varphi$ at $x_{0}$, induces an isomorphism

$$
\operatorname{incl}_{*}: \Omega_{m-n}^{\mathrm{fr}}\left(\Lambda\left(N, y_{0}\right)\right) \stackrel{\cong}{\longrightarrow} \Omega_{m-n}(E(f) ; \widetilde{\varphi}) .
$$

The framed bordism class

$$
\operatorname{incl}_{*}^{-1}\left(\widetilde{\omega}_{\varepsilon}(f)\right) \in \Omega_{m-n}^{\mathrm{fr}}\left(\Lambda\left(N, y_{0}\right)\right), \quad \varepsilon=+ \text { or }-,
$$

is invariant under base point preserving link homotopies.

Proof. Our choices (including those which are incorporated in 3.1) induce isomorphisms

$$
\Omega_{m-n}^{\mathrm{fr}}\left(\Lambda\left(N, y_{0}\right)\right) \cong \Omega_{m-n}^{\mathrm{fr}}\left(\operatorname{pr}^{-1}\left(x_{0}\right)\right) \cong \Omega_{m-n}\left(\operatorname{pr}^{-1}\left(x_{0}\right) ; \widetilde{\varphi} \mid\right)
$$

and so does the fiber inclusion; this follows via a cell-by-cell argument applied to (projected) maps into $M$.

Similarly if $F$ is a base point preserving link homotopy, the (generic) coincidence manifold $C_{\varepsilon}(F) \subset M \times I$ can be retracted to $\left\{x_{0}\right\} \times I$ and hence yields the required bordism in the fiber $\operatorname{pr}^{-1}\left(x_{0}\right) \sim \Lambda\left(N, y_{0}\right)$.

REMARK 3.9. If $n>0$, then every (free) link homotopy class can be represented by a base point preserving link map $f$ (i.e. $f_{1}\left(x_{01}\right) \neq f_{2}\left(x_{02}\right)$ are fixed preassigned points in $N \times \mathbb{R}$ ). 
If two such link maps $f$ and $\widehat{f}$ are related by a free link homotopy $F$, then we deduce (under the assumptions of Proposition 3.8 and provided $\left.m_{1}, m_{2} \leq n-2\right)$ that

$$
\operatorname{incl}_{*}^{-1}\left(\widetilde{\omega}_{\varepsilon}(\widehat{f})\right)=\operatorname{sign}\left(\tau_{1}\right) \cdot \operatorname{sign}\left(\tau_{2}\right) \cdot c\left(\tau_{1}, \tau_{2}\right)_{*}\left(\operatorname{incl}_{*}^{-1}\left(\widetilde{\omega}_{\varepsilon}(f)\right)\right),
$$

where $\tau_{i}=F_{i}^{\prime}\left(x_{0 i},-\right)$ denotes the loop traced out in $N$ during the homotopy, $i=1,2$, and the self-homotopy equivalence $c\left(\tau_{1}, \tau_{2}\right)$ of $\Lambda\left(N, y_{0}\right)$ is defined by

$$
c\left(\tau_{1}, \tau_{2}\right)(\varrho)=\sigma_{1} \tau_{1}^{-1} \sigma_{1}^{-1} \varrho \sigma_{2} \tau_{2} \sigma_{2}^{-1}, \quad \varrho \in \Lambda\left(N, y_{0}\right) ;
$$

moreover, $\operatorname{sign}\left(\tau_{i}\right)$ equals +1 or -1 according as $\tau_{i}$ preserves the orientation of $N$ or not.

4. Products of spheres. In this section we consider the special case

$$
N=\left(S^{1}\right)^{q} \times S^{2} \times \cdots \times S^{r_{l}}=\left(S^{1}\right)^{q} \times N^{\prime},
$$

where

$$
N^{\prime}=\prod_{i=1}^{l} S^{r_{i}}, \quad 2 \leq r_{1} \leq r_{2} \leq \cdots \leq r_{l}, 1 \leq l<\infty,
$$

denotes the subproduct formed by the simply connected factor spheres. Pick base points $*_{1}=1 \in S^{1} ; *_{r_{i}} \in S^{r_{i}}, i=1, \ldots, l ; y_{0}^{\prime}=\left(*_{r_{1}}, \ldots, *_{r_{l}}\right) \in N^{\prime}$ and $y_{0}=\left(1, \ldots, 1, y_{0}^{\prime}\right) \in N$. In view of Proposition 3.8 the following sample calculation frequently determines the group in which our $\widetilde{\omega}$-invariants lie.

THEOREM 4.1. There are canonical isomorphisms of $\Omega_{*}^{\mathrm{fr}}$-modules

$$
\Omega_{*}^{\mathrm{fr}}\left(\Lambda\left(N, y_{0}\right)\right) \cong \bigoplus_{j \in \mathbb{Z}^{q}} \Omega_{*}^{\mathrm{fr}}\left(\Lambda\left(N^{\prime}, y_{0}^{\prime}\right)\right)
$$

and

$$
h=\bigoplus h_{k}: \Omega_{*}^{\mathrm{fr}}\left(\Lambda\left(N^{\prime}, y_{0}^{\prime}\right)\right) \stackrel{\cong}{\longrightarrow} \bigoplus_{k \in \mathbb{N}^{l}} \Omega_{*-d(k)}^{\mathrm{fr}},
$$

where $d(k):=\sum_{i=1}^{l} k_{i}\left(r_{i}-1\right)$ for $k=\left(k_{1}, \ldots, k_{l}\right) \in \mathbb{N}^{l}$ and $\mathbb{N}$ denotes the set of natural numbers including 0 .

Thus the Pontryagin-Thom isomorphism $\Omega_{*}^{\mathrm{fr}} \cong \pi_{*}^{S}$ makes the framed bordism groups of the loop space of $N$ accessible to standard methods of stable homotopy theory.

Proof. Consider $\mathbb{Z}^{q}$ as a discrete topological subspace of $\mathbb{R}^{q}$ and apply the exponential map $\mathbb{R}^{q} \rightarrow\left(S^{1}\right)^{q}$ to the straight path in $\mathbb{R}^{q}$ joining 0 to any point in $\mathbb{Z}^{q}$. This yields the homotopy equivalences $\mathbb{Z}^{q} \sim \Lambda\left(\left(S^{1}\right)^{q}\right)$ and

$$
\Lambda\left(N, y_{0}\right) \sim \mathbb{Z}^{q} \times \Lambda\left(N^{\prime}, y_{0}^{\prime}\right)
$$

as well as the first isomorphism claimed above. 
The second isomorphism generalizes a geometric construction which was discussed in detail in [K5] and which is closely related to James-Hopf invariants.

Given a framed bordism class $\nu$ of $\Lambda\left(N^{\prime}, y_{0}^{\prime}\right)$, consider the adjoint

$$
v=\left(v_{1}, \ldots, v_{l}\right):(V \times \mathbb{R} \cup\{\infty\}, \infty) \rightarrow\left(N^{\prime}=S^{r_{1}} \times \ldots \times S^{r_{l}}, y_{0}^{\prime}\right)
$$

of a generic representative. Then for $i=1, \ldots, l$ the inverse image $W_{i}:=$ $v_{i}^{-1}\left(*_{i}^{\prime}\right)$ of a point $*_{i}^{\prime} \neq *_{r_{i}}$ in $S^{r_{i}}$ is a smoothly embedded framed submanifold of $V \times \mathbb{R}$. As in $[\mathrm{K} 5, \S 8]$ (see also [KS]) we may even assume that this embedding projects to a generic framed $\left(r_{i}-1\right)$-codimensional immersion $e_{i}^{\prime}$ into $V$. Its $k_{i}$-fold self-intersection yields a $k_{i}\left(r_{i}-1\right)$-codimensional immersion $e_{i}^{k_{i}}$ which is again framed since the intersection branches are ordered by the $\mathbb{R}$-component of the embedding $W_{i} \subset V \times \mathbb{R}$.

Now for $k=\left(k_{1}, \ldots, k_{l}\right) \in \mathbb{N}^{l}$ define $h_{k}(\nu)$ to be the framed bordism class of the transverse intersection of the immersions $e_{1}^{k_{1}}, \ldots, e_{l}^{k_{l}}$ in $V$. A straightforward generalization of the proofs in $[\mathrm{K} 5, \S 8]$ (where the case $l=1$ was discussed) shows that $h_{k}$ is well defined and $h=\bigoplus h_{k}$ is bijective.

REMARK 4.2. The theorem above leads to an interpretation in terms of Laurent polynominals:

$$
\begin{aligned}
\Omega_{*}^{\mathrm{fr}}\left(\Lambda\left(N, y_{0}\right)\right) & \cong \bigoplus_{\left(j_{1}, \ldots, j_{q}\right) \in \mathbb{Z}^{q}} \Omega_{*}^{\mathrm{fr}}\left(\Lambda\left(N^{\prime}, y_{0}^{\prime}\right)\right) X_{1}^{j_{1}} \cdots X_{q}^{j_{q}} \\
& =\Omega_{*}^{\mathrm{fr}}\left(\Lambda\left(N^{\prime}, y_{0}^{\prime}\right)\right)\left[X_{1}^{ \pm 1}, \ldots, X_{q}^{ \pm 1}\right] .
\end{aligned}
$$

Expressed in this language the operation $c\left(\tau_{1}, \tau_{2}\right)_{*}$ discussed in Remark 3.9 amounts just to multiplication by a fixed monomial $X_{1}^{j_{1}} \cdots X_{q}^{j_{q}}$. Thus we conclude (under the assumptions of Proposition 3.8 and provided $m_{1}, m_{2} \leq$ $n-2)$ that $\operatorname{incl}_{*}^{-1}\left(\widetilde{\omega}_{\varepsilon}(f)\right)$, considered up to multiplication by such monomials, is invariant under base point free link homotopies.

(Note the analogy to Alexander polynomials.)

5. Spherical link maps. In this section we discuss the case where $M_{i}$ is the sphere $S^{m_{i}}$, equipped with a base point $x_{0 i}, i=1,2$. Also choose base points $y_{01} \neq y_{02}$ and $y_{0}$ in $N$ and paths $\sigma_{1}$ and $\sigma_{2}$ in $N$ joining $y_{0}$ to $y_{01}$ and $y_{02}$, respectively. Let $\mathrm{BLM}_{m_{1}, m_{2}}(N \times \mathbb{R})$ denote the set of base point preserving link homotopy classes of base point preserving link maps

$$
f=f_{1} \amalg f_{2}: S^{m_{1}} \amalg S^{m_{2}} \rightarrow N \times \mathbb{R}
$$

(i.e. $\left.f_{i}\left(x_{0 i}\right)=\left(y_{0 i}, 0\right)\right)$, and define

$$
\mathrm{BLM}^{(i)}:=\left\{[f] \in \mathrm{BLM}_{m_{1}, m_{2}}(N \times \mathbb{R}) \mid 0=\left[f_{i}\right] \in \pi_{m_{i}}(N \times \mathbb{R})\right\},
$$


Assume $1 \leq m_{1}, m_{2} \leq n-2$ throughout this section. Then link maps as well as link homotopies can be deformed until the $i$ th component avoids $\left\{y_{0 i \pm 1}\right\} \times \mathbb{R} \subset N \times \mathbb{R}, i=1,2$. Thus we can add two link maps $f$ and $\widehat{f}$ by "stacking them on top of each other" with respect to the height given by the $\mathbb{R}$-component in $N \times \mathbb{R}$ : shift the link map $\widehat{f}$ in the positive $\mathbb{R}$-direction until it is entirely above $f$ and join base points along $\left\{y_{0 i}\right\} \times \mathbb{R}$. The resulting addition makes $\mathrm{BLM}_{m_{1}, m_{2}}(N \times \mathbb{R})$ into a semigroup with null element.

According to Proposition 3.8 our choices determine isomorphisms allowing us to identify our $\widetilde{\omega}$-invariants with elements in a fixed group which does not vary with $f$ any more (for a more explicit description see the proof below). This greatly simplifies statements about link homotopy invariance, additivity, value sets etc.

Proposition 5.2. Assume that $1 \leq m_{1}, m_{2} \leq n-2$. Then for $\varepsilon=+$ and $\varepsilon=-$ the $\widetilde{\omega}_{\varepsilon}$-obstruction determines a well defined map

$$
\widetilde{\omega}_{\varepsilon}: \operatorname{BLM}_{m_{1}, m_{2}}(N \times \mathbb{R}) \rightarrow \Omega_{m-n}^{\mathrm{fr}}\left(\Lambda\left(N, y_{0}\right)\right) .
$$

For all $[f],[\widehat{f}] \in \mathrm{BLM}_{m_{1}, m_{2}}(N \times \mathbb{R})$ we have

$$
\begin{aligned}
& \widetilde{\omega}_{+}([f]+[\widehat{f}])=\widetilde{\omega}_{+}([f])+\widetilde{\omega}_{+}([\widehat{f}])+\widetilde{\omega}\left(f_{1}^{\prime} \circ p_{1}, \widehat{f}_{2}^{\prime} \circ p_{2}\right), \\
& \widetilde{\omega}_{-}([f]+[\widehat{f}])=\widetilde{\omega}_{-}([f])+\widetilde{\omega}_{-}([\widehat{f}])+\widetilde{\omega}\left(\widehat{f}_{1}^{\prime} \circ p_{1}, f_{2}^{\prime} \circ p_{2}\right),
\end{aligned}
$$

where the last summand denotes the coincidence invariant (compare 2.9 and $[\mathrm{K} 5,4.4]$ and 2.4) of the indicated projections to $N$.

In particular, $\widetilde{\omega}_{\varepsilon} \mid \mathrm{BLM}^{(i)}$ is a homomorphism of semigroups; its image is a group and is invariant under both the left and the right action of $\pi_{1}(N)$ on $\Omega_{m-n}^{\mathrm{fr}}\left(\Lambda\left(N, y_{0}\right)\right), i=1,2$.

Proof. Let us first specify a representative $\widetilde{g}_{\varepsilon}: C_{\varepsilon}(f) \rightarrow \Omega_{m-n}\left(\Lambda\left(N, y_{0}\right)\right)$ of $\widetilde{\omega}_{\varepsilon}(f)$. Since $m-n+1<m_{i}$ the projection of $C_{\varepsilon}(f) \subset S^{m_{1}} \times S^{m_{2}}$ to $S^{m_{i}}$ generically avoids the (antipodal) point $-x_{0 i}$ and hence allows a retraction $\varrho_{i}$ in $S^{m_{i}}$ to the base point $x_{0 i}, i=1,2$. For any $x \in C_{\varepsilon}(f)$ the loop $\widetilde{g}_{\varepsilon}(x)$ can then be described as the composite of paths

$$
y_{0} \stackrel{\sigma_{1}}{\longrightarrow} y_{01} \stackrel{f_{1}^{\prime}\left(\varrho_{1}(x,-)\right)^{-1}}{\longrightarrow} f_{1}^{\prime}(x)=f_{2}^{\prime}(x) \stackrel{f_{2}^{\prime} \varrho_{2}(x,-)}{\longrightarrow} y_{02} \stackrel{\sigma_{2}^{-1}}{\longrightarrow} y_{0} .
$$

If we compose $f$ with a base point preserving reflection $r$ of $S^{m_{1}}$ or $S^{m_{2}}$ we change the framing of $C_{\varepsilon}(f)$ and its location in $S^{m_{1}} \times S^{m_{2}}$ but not the homotopy class of $\tilde{g}_{\varepsilon}$. Thus

$$
\widetilde{\omega}_{\varepsilon}\left(f_{1} \circ r \amalg f_{2}\right)=\widetilde{\omega}_{\varepsilon}\left(f_{1} \amalg f_{2} \circ r\right)=-\widetilde{\omega}_{\varepsilon}(f) .
$$

If $\tau_{i}$ is a loop in $N$ starting and ending at $x_{0 i}$ (and generically avoiding $f_{i \pm 1}$ ) and we use it to modify $f_{i}$ via the standard operation, we conclude for the resulting link map that

$$
\widetilde{\omega}_{\varepsilon}\left(\tau_{1 *}\left(f_{1}\right) \amalg \tau_{2 *}\left(f_{2}\right)\right)= \pm\left(\sigma_{1} \tau_{1}^{-1} \sigma_{1}^{-1}\right) \widetilde{\omega}_{\varepsilon}(f)\left(\sigma_{2} \tau_{2} \sigma_{2}^{-1}\right) .
$$


Finally note that the overcrossing locus $C_{\varepsilon}(f+\widehat{f})$ consists of $C_{\varepsilon}(f) \cup$ $C_{\varepsilon}(\widehat{f})$ and of the full coincidence locus of $\left(f_{1}^{\prime}, \widehat{f}_{2}^{\prime}\right)$. The proposition follows.

Next consider the invariant

$$
\widetilde{\widetilde{\omega}}_{\varepsilon}: \operatorname{BLM}_{m_{1}, m_{2}}(N \times \mathbb{R}) \rightarrow \Omega_{m-n}^{\mathrm{fr}}\left(\Lambda\left(N, y_{0}\right)\right) \times \bigoplus_{i=1}^{2} \pi_{m_{i}}\left(N, y_{0 i}\right)
$$

which enriches $\widetilde{\omega}_{\varepsilon}(f)$ by the homotopy classes $\left[f_{1}^{\prime}\right]$ and $\left[f_{2}^{\prime}\right]$ of the component maps $f_{1}$ and $f_{2}$ of $f$. In view of $2.9, \widetilde{\omega}_{+}$determines $\widetilde{\widetilde{\omega}}_{-}$.

Corollary 5.5. For both $\varepsilon=+$ and $\varepsilon=-$ we have

$$
\widetilde{\widetilde{\omega}}_{\varepsilon}\left(\mathrm{BLM}_{m_{1}, m_{2}}(N \times \mathbb{R})\right)=\widetilde{\omega}_{\varepsilon}\left(\mathrm{BLM}^{(1)} \cap \mathrm{BLM}^{(2)}\right) \times \bigoplus_{i=1}^{2} \pi_{m_{i}}\left(N, y_{0 i}\right)
$$

(compare 5.1).

Proof. Given $i=1,2$, let

$$
c_{i}: \pi_{m_{i}}\left(N, y_{0 i}\right) \cong \pi_{m_{i}}\left(N-\left\{y_{0 i \pm 1}\right\}, y_{0 i}\right) \rightarrow \mathrm{BLM}^{(i)}
$$

be defined by adding the constant map with value $y_{0 i \pm 1} \in N=N \times\{0\} \subset$ $N \times \mathbb{R}$. Then we have the bijection

$$
\left(\mathrm{BLM}^{(1)} \cap \mathrm{BLM}^{(2)}\right) \times \bigoplus_{i=1}^{2} \pi_{m_{i}}\left(N, y_{0 i}\right) \rightarrow \mathrm{BLM}_{m_{1}, m_{2}}(N \times \mathbb{R}),
$$

$\left(f,\left[\widehat{f}_{1}\right],\left[\widehat{f}_{2}\right]\right) \mapsto c_{2}\left(\left[\widehat{f}_{2}\right]\right)+f+c_{1}\left(\left[\widehat{f}_{1}\right]\right)$. Here the order of summation is chosen in such a way that according to 5.2 the sum has the same $\widetilde{\omega}_{+}$-value as $f$. The corollary follows.

Proof of Theorem 1.1 of the introduction. Clearly the assertion holds if $m_{1}=0$.

Thus assume that $m_{1} \geq 1$. Then also $m_{1}, m_{2} \leq n-2$. In view of the decomposition 5.6 we have to prove our claim only for $f, \widehat{f} \in \mathrm{BLM}^{(1)} \cap$ $\mathrm{BLM}^{(2)}$ with $\widetilde{\omega}_{+}(f)=\widetilde{\omega}_{+}(\widehat{f})$. Put $(-\widehat{f}):=\widehat{f}_{1} \circ r \amalg \widehat{f}_{2}$ (cf. 5.3). Then according to 5.2 we have

$$
\widetilde{\omega}_{+}(f+(-\widehat{f}))=\widetilde{\omega}_{+}(f)-\widetilde{\omega}_{+}(\widehat{f})=0 .
$$

Thus it follows from Theorem 2.13 that $f+(-\widehat{f})$ (and similarly $(-\widehat{f})+\widehat{f}$ ) is link nulhomotopic. Therefore

$$
f \sim f+((-\widehat{f})+\widehat{f}) \sim(f+(-\widehat{f}))+\widehat{f} \sim \widehat{f} .
$$

Finally, we discuss a simple construction which produces many link maps with interesting $\widetilde{\omega}_{ \pm}$-invariants.

Consider the case where $N=N_{1} \times N_{2}$ is the product of two manifolds of dimensions $n_{1}, n_{2} \geq 1$. Then the base points take the form $y_{0 i}=\left(y_{0 i}^{1}, y_{0 i}^{2}\right)$, $i=1,2$, and we may assume that $y_{01}^{1} \neq y_{02}^{1}$ and $y_{01}^{2} \neq y_{02}^{2}$. 
Also consider the link map

$$
e=e_{1} \amalg e_{2}: S^{n_{1}} \amalg N_{2} \rightarrow N \times \mathbb{R}
$$

formed by the embedding

$$
e_{2}: N_{2} \cong\left\{y_{02}^{1}\right\} \times N_{2} \times\{0\} \subset N \times \mathbb{R}
$$

and by a meridian (of radius $\varrho$, say) which is based at $\left(y_{01}, 0\right)$ and which lies in $N_{1} \times\left\{y_{01}^{2}\right\} \times \mathbb{R}$ (so that $\left(y_{02}^{1}, y_{01}^{2}\right)$ is the only over/undercrossing point). Note that $e_{1}$ can be contracted within a normal ball of $e_{2}$ with center at $\left(y_{02}^{1}, y_{01}^{2}, 0\right)$.

Thus composition with $e$ yields a map

$$
\pi_{m_{1}}\left(S^{n_{1}}\right) \times \pi_{m_{2}}\left(N_{2}\right) \rightarrow \mathrm{BLM}^{(1)}
$$

(compare 5.1). Moreover the inclusion $e_{2}^{\prime}$ induces a monomorphism

$$
e_{2 *}^{\prime}: \Omega_{*}^{\mathrm{fr}}\left(\Lambda\left(N_{2}, y_{02}^{2}\right)\right) \rightarrow \Omega_{*}^{\mathrm{fr}}\left(\Lambda\left(N, y_{0}\right)\right)
$$

Proposition 5.9. Assume $1 \leq m_{1}, m_{2} \leq n_{1}+n_{2}-2$. Then for all $\left[f_{1}\right] \in \pi_{m_{1}}\left(S^{n_{1}}\right),\left[f_{2}\right] \in \pi_{m_{2}}\left(N_{2}\right)$ and for both $\varepsilon=+$ and $\varepsilon=-$ we have

$$
\widetilde{\omega}_{\varepsilon}\left(e_{1} \circ f_{1} \amalg e_{2} \circ f_{2}\right)= \pm \operatorname{deg}\left(f_{1}\right) \cdot e_{2 *}^{\prime}\left(\widetilde{\operatorname{deg}}\left(f_{2}\right)\right),
$$

where the degrees

$$
\begin{aligned}
& \operatorname{deg}\left(f_{1}\right):=\omega\left(f_{1}, *\right) \in \Omega_{m_{1}-n_{1}}^{\mathrm{fr}}, \\
& \widetilde{\operatorname{deg}}\left(f_{2}\right):=\widetilde{\omega}\left(f_{2}, y_{01}^{2}\right) \in \Omega_{m_{2}-n_{2}}^{\mathrm{fr}}\left(\Lambda\left(N_{2}, y_{02}^{2}\right)\right)
\end{aligned}
$$

measure coincidences with the indicated constant maps (as in [K5, 1.11, 1.12, and 7.5]).

Proof. Define $L_{1}:=f_{1}^{-1}\left(\left\{\left(y_{02}^{1},-\varepsilon \varrho\right)\right\}\right)$ and $L_{2}:=f_{2}^{-1}\left(\left\{y_{01}^{2}\right\}\right)$. Then the over/undercrossing locus is

$$
C_{\varepsilon}=L_{1} \times L_{2} \subset S^{m_{1}} \times S^{m_{2}} .
$$

The corresponding loops are described as in the proof of Proposition 5.2: apply $f_{i}$ to the paths resulting from a contraction of $L_{i}, i=1,2$. Since $f_{1}$ is nulhomotopic, the framed manifold $L_{1}$ is equipped with a trivial map and contributes to $\widetilde{\omega}_{\varepsilon}$ only via the $\Omega_{*}^{\mathrm{fr}}$-module structure on $\Omega_{*}^{\mathrm{fr}}\left(\Lambda\left(N, y_{0}\right)\right)$.

Often it is convenient to identify the framed bordism ring $\Omega_{*}^{\mathrm{fr}}$ with the stable homotopy $\operatorname{ring} \pi_{*}^{S}$ of spheres via the Pontryagin-Thom isomorphism. Then $\operatorname{deg}\left(f_{1}\right)$ corresponds to the stable Freudenthal suspension $\pm E^{\infty}\left(\left[f_{1}\right]\right)$.

COROLlary 5.10. For both $\varepsilon=+$ and $\varepsilon=-$ the value set $\widetilde{\omega}_{\varepsilon}\left(\mathrm{BLM}^{(1)}\right)$ (cf. 5.1 and 5.2) contains at least the subgroup generated by the set

$$
\left.E^{\infty}\left(\pi_{m_{1}}\left(S^{n_{1}}\right)\right) \cdot e_{2 *}^{\prime} \widetilde{(\operatorname{deg}}\left(\pi_{m_{2}}\left(N_{2}\right)\right)\right) \subset \Omega_{m-n}^{\mathrm{fr}}\left(\Lambda\left(N, y_{0}\right)\right)
$$

and by the left and right group action of $\pi_{1}\left(N, y_{0}\right)$. 
This follows from Propositions 5.2 and 5.9.

EXAMPLE 5.11 (Spherical link maps into products of spheres). Let

$$
N=\left(S^{1}\right)^{q} \times S^{2} \times \ldots \times S^{r_{l}}
$$

be as in Section 4. Given $1 \leq i \leq l$, let $N_{2}$ and $N_{1}$ be the $i$ th higherdimensional sphere $S^{r_{i}}$ (i.e. $r_{i} \geq 2$ ) and the product of the remaining factors, resp. Also recall from Theorem 1.14 in [K5] that $\widetilde{\operatorname{deg}}\left(\pi_{m_{2}}\left(S^{r_{i}}\right)\right)$ corresponds to the image of the total stabilized James-Hopf invariant homomorphism

$$
\Gamma_{i}=\bigoplus_{k \geq 0} E^{\infty} \circ \gamma_{k+1}: \pi_{m_{2}}\left(S^{r_{i}}\right) \rightarrow \bigoplus_{k \geq 0} \pi_{m_{2}-r_{i}-k\left(r_{i}-1\right)}^{S} .
$$

Thus according to Corollary 5.10 at least all the elements of the subgroup

$$
\bigoplus_{j \in \mathbb{Z}^{q}} E^{\infty}\left(\pi_{m_{1}}\left(S^{n-r_{i}}\right)\right) \cdot \Gamma_{i}\left(\pi_{m_{2}}\left(S^{r_{i}}\right)\right)
$$

of

$$
\Omega_{m-n}^{\mathrm{fr}}\left(\Lambda\left(N, y_{0}\right)\right) \cong \bigoplus_{j \in \mathbb{Z}^{q}} \bigoplus_{k \in \mathbb{N}^{l}} \pi_{m-n-d(k)}^{S}
$$

(cf. Theorem 4.1) occur as values of our invariants $\widetilde{\omega}_{+}$and $\widetilde{\omega}_{-}$for suitable link maps, $i=1, \ldots, l$.

SpeCial CASE 5.11a: $N=S^{2} \times S^{3}, m_{1}=m_{2}=3$. Here $\widetilde{\omega}_{+}$and $\widetilde{\omega}_{-}$take values in

$$
\Omega_{1}^{\mathrm{fr}}(\Lambda N) \cong \Omega_{1}^{\mathrm{fr}}\left(\Lambda S^{2}\right) \stackrel{\cong}{\stackrel{\cong}{h}} \Omega_{1}^{\mathrm{fr}} \oplus \Omega_{0}^{\mathrm{fr}}=\mathbb{Z}_{2} \oplus \mathbb{Z}
$$

(cf. 4.1). If we pick $N_{2}=S^{2}$ and $N_{1}=S^{3}$ we see that

$$
(1,1)=\left(E^{\infty}, \text { Hopf invariant }\right)(\text { Hopf map })=\Gamma_{1}(\text { Hopf map })
$$

corresponds to a value of $\widetilde{\omega}_{ \pm}$; if we pick $N_{2}=S^{3}, N_{1}=S^{2}$ we obtain the same for $\left(1=E^{\infty}\right.$ (Hopf map), 0$)$. Thus the invariant $\widetilde{\omega}_{ \pm}$(even when restricted to $\mathrm{BLM}^{(i)}, i=1$ or 2 , cf. 5.1$)$ assumes all possible values in its target group.

Both the invariant $\omega_{+}$(cf. 2.10) and the Pilz invariant $\alpha_{w}$ (cf. 3.7) capture only the $\mathbb{Z}_{2}$-component of $\widetilde{\omega}_{+}$, i.e. the framed bordism class of the overcrossing locus $C_{+}$(without any nontrivial map into a target space or any nontrivial decomposition into Nielsen classes).

Special CASE 5.11b: $N=\left(S^{1}\right)^{3} \times S^{2}, m_{1}=m_{2}=3$. By Theorem 4.1 the target group of $\widetilde{\omega}_{ \pm}$is as stated in Example 1.3 of the introduction. Moreover, every element $(1,1)_{j}, j \in \mathbb{Z}^{3}$, lies in $\widetilde{\omega}_{ \pm}\left(\mathrm{BLM}^{(i)}\right), i=1,2$ (cf. 5.1, 5.2, and 5.11a). So also does $(1,0)_{j}, j \in \mathbb{Z}^{3}$. Indeed, the Fenn-Rolfsen link map $f_{\mathrm{FR}}: S^{2} \amalg S^{2} \rightarrow \mathbb{R}^{4}$ (cf. [FR]) can be suspended with respect to each component to yield a link map $\Sigma_{1}\left(\Sigma_{2}\left(f_{\mathrm{FR}}\right)\right): S^{3} \amalg S^{3} \rightarrow \mathbb{R}^{6}$ with nontrivial $\alpha$-invariant (cf. e.g. [K2, 2.1, 2.10, and 4.8]); included in a small ball in $N \times \mathbb{R}$ 
and connected to the base points in a suitable way this yields a link map in $\mathrm{BLM}^{(1)} \cap \mathrm{BLM}^{(2)}$ with the desired $\widetilde{\omega}_{ \pm}$-value. Thus

$$
\widetilde{\omega}_{\varepsilon}\left(\mathrm{BLM}^{(i)}\right)=\bigoplus_{j \in \mathbb{Z}^{3}}\left(\mathbb{Z}_{2} \oplus \mathbb{Z}\right)
$$

for every combination of $i=1,2$ and $\varepsilon=+$ or - .

In this special case the Pilz invariant $\alpha_{w} \in \bigoplus \mathbb{Z}_{2}$ is obtained by projecting to the $\mathbb{Z}_{2}$-factors. It is much stronger here than the invariant $\omega_{+} \in \mathbb{Z}_{2} \cong$ $\Omega_{1}^{\mathrm{fr}} \cong \Omega_{1}^{\mathrm{fr}}\left(S^{3} \times S^{3}\right)$ which measures just the framed bordism class of the overcrossing locus without retaining its Nielsen decomposition.

On the other hand in some situations Nielsen decompositions are irrelevant and $\omega_{+}$captures more information than the Pilz invariant $\alpha_{w}$.

EXAMPLE 5.13. Given an integer $s \geq 1$, equip $S^{s}=\partial\left(B^{s+1}\right)$ with the boundary framing inherited from the $(s+1)$-ball and consider the link map

$$
f=f_{1} \amalg f_{2}: S^{3} \times S^{s} \amalg S^{3} \rightarrow S^{2} \times S^{3} \times \mathbb{R}
$$

defined by $f_{1}\left(x_{1}, x_{2}\right):=\left((\operatorname{Hopf} \operatorname{map})\left(x_{1}\right), *_{S^{3}}, 0\right)$ for $\left(x_{1}, x_{2}\right) \in S^{3} \times S^{s}$ and $f_{2}(x):=\left(*_{S^{2}}, x, 1\right)$ for $x \in S^{3}$. Then $\alpha_{w}(f)=0$ while $\omega_{+}(f)$ is nontrivial. Indeed, the coincidence locus $C_{+}=S^{1} \times S^{s}$ is framed nullbordant, but

$$
\omega_{+}(f)=\left[C_{+}, g_{+}, \bar{g}_{+}\right] \in \Omega_{s+1}^{\mathrm{fr}}\left(S^{3} \times S^{s} \times S^{3}\right) .
$$

projects to

$$
(0,1) \in \Omega_{s+1}^{\mathrm{fr}}\left(S^{s}\right) \cong \Omega_{s+1}^{\mathrm{fr}} \oplus \Omega_{1}^{\mathrm{fr}}
$$

(compare 2.15).

\section{References}

[BGZ] S. Bogatyı̆, D. Gonçalves, and H. Zieschang, Coincidence theory: the minimizing problem, Proc. Steklov Inst. Math. 225 (1999), 45-77.

[B] R. Brown, Nielsen fixed point theory on manifolds, in: Nielsen Theory and Reidemeister Torsion, J. Jezierski (ed.), Banach Center Publ. 49, Inst. Math. Polish Acad. Sci., 1999, 19-27.

[FR] R. Fenn and D. Rolfsen, Spheres may link homotopically in 4-space, J. London Math. Soc. 34 (1986), 177-184.

[HK] N. Habegger and U. Kaiser, Link homotopy in the 2-metastable range, Topology 37 (1998), 75-94.

[HQ] A. Hatcher and F. Quinn, Bordism invariants of intersections of submanifolds, Trans. Amer. Math. Soc. 200 (1974), 327-344.

[J ] J. Jezierski, On generalizing the Nielsen coincidence theory to non-oriented manifolds, in: Nielsen Theory and Reidemeister Torsion, J. Jezierski (ed.), Banach Center Publ. 49, Inst. Math. Polish Acad. Sci., 1999, 189-202.

[Ka] U. Kaiser, Link Theory in Manifolds, Lecture Notes in Math. 1669, Springer, 1997. 
[K1] U. Koschorke, Vector Fields and Other Vector Bundle Monomorphisms - A Singularity Approach, Lecture Notes in Math. 847, Springer, 1981.

[K2] - Link maps and the geometry of their invariants, Manuscripta Math. 61 (1988), 383-415.

[K3] - Link maps in arbitrary manifolds and their homotopy invariants, J. Knot Theory Ramifications 12 (2003), 79-104.

[K4] - Selfcoincidences in higher codimensions, J. Reine Angew. Math. 576 (2004), $1-10$.

[K5] - Nielsen coincidence theory in arbitrary codimensions, preprint, Siegen, 2003; see http://www.math.uni-siegen.de/topology/publications.html.

[KS] U. Koschorke and B. Sanderson, Self-intersections and higher Hopf invariants, Topology 17 (1978), 283-290.

[M] J. Milnor, Link groups, Ann. of Math. 59 (1954), 177-195.

[P] A. Pilz, Two-component link maps in manifolds, doctoral dissertation, Siegen, 2004 .

[S] G. Scott, Homotopy links, Abh. Math. Sem. Univ. Hamburg 32 (1968), 186-190.

[T] H. Toda, Composition Methods in Homotopy Groups of Spheres, Princeton Univ. Press, 1962.

Universität Siegen

Emmy Noether Campus

Walter-Flex-Str. 3

D-57068 Siegen, Germany

E-mail: koschorke@mathematik.uni-siegen.de

Received 10 April 2004;

in revised form 15 July 2004 